\title{
Mémoire de l'Institut forestier du Canada, section Champlain présenté à la Commission sur la Protection des Forêts concernant la stratégie de protection des forêts
}

\author{
Juin 1991
}

\section{L'Institut forestier du Canada}

L'Institut Forestier du Canada (I.F.C.) est un organisme canadien, à but non-lucratif et bénévole. Sa mission est de faire progresser la gestion des ressources forestières du Canada, assumer un leadership en foresterie, et développer la sensibilisation du public sur les actualités forestières canadiennes et internationales. Nos objectifs sont:

(a) d'améliorer la foresterie au Canada sous tous ses aspects;

(b) de sensibiliser davantage le public à la foresterie;

(c) d'aider nos membres à acquérir plus de compétences et d'esprit de corps et;

(d) de collaborer avec les autres organisations dont les soucis et les intérêts sont semblables aux nôtres.

L'institut Forestier du Canada se subdivise en 22 sections régionales. La région métropolitaine est comprise à l'intérieur du territoire couvert par la section Champlain. Ce territoire comprend également la grande région de l'Estrie. Cette décentralisation permet à chaque section d'organiser ses propres activités, de participer à des programmes de sensibilisation du public et la préparation d'exposés qui reflètent le contexte socio-économique particulier à chaque région.

Le conseil de la section Champlain est composé de membres occupant des emplois au sein de l'industrie forestière, de la forêt privée, de différents paliers de gouvernements, de maisons d'enseignements et de firmes de consultats.

Conformément à la mission qu'elle s'est dotée, la section Champlain participe à ces audiences publiques afin d'atteindre une orientation équilibrée et progressive, une planification et une gestion intelligente ainsi que l'utilisation variée et coordonnée des terres forestières québécoises.

\section{Objectifs poursuivis par la Stratégie}

Le monde forestier ne peut qu'être satisfait de la philosophie utilisée par le Ministère des Forêts dans son projet de stratégie de protection des forêts. Nous sommes en mesure d'y percevoir une volonté de gérer les ressources forestières dans un esprit de développement durable.

Cette stratégie s'aligne dans le sens de l'orientation globale de la loi 150 en poursuivant le changement. Les tendances d'exploitation des ressources forestières cèdant le pas à des concepts d'aménagement. Les générations futures verront finalement l'utilisation globale du milieu forestier et non seulement l'exploitation de la matière ligneuse.

Cette stratégie poursuit aussi les objectifs sociaux actuels en tâchant de réduire les impacts de l'utilisation de milieu forestier. Les Québécois sont de plus en plus conscients de l'importance qu'on doit accorder à notre environnement et désirent améliorer ce qui était hier des pratiques courantes.

\section{Politique d'utilisation du territoire}

Face aussi à l'immensité du territoire québécois, nous sommes d'avis qu'il aurait fallu identifier les zones forestières économiquement propices à la production de matière ligneuse et d'assigner à ces zones une vocation industrielle. Il ne faut pas oublier qu'à défaut de maximiser le rendement du capital ligneux, nous courrons le risque au cours des prochaines décennies d'avoir à entamer le capital pour permettre la survie de plusieurs agglomérations, ou pire de voir l'exode des populations vers les centres urbains s'accentuer.

Il nous faut à très brève échéance attribuer au domaine forestier une vocation en fonction de la capacité du milieu, et ne retenir qu'à des fins d'aménagement intensif que les territoires à haut potentiel de rendement en matière ligneuse. Les territoires restant auront été désignés comme ayant une autre vocation ou un potentiel forestier secondaire, sur lesquels sera pratiqué un aménagement forestier extensif que visera à maintenir les peuplements forestiers en équilibre selon leur évolution écologique normale.

Il sera donc possible d'augmenter la superficie des territoires dont la population souhaite en tirer une vocation polyvalente et intégrée, soit récréative, esthétique ou faunique, sans oublier la capacité du milieu à devenir des usines de transformation des gaz à effet de serre, et sans mettre en péril la survie des fonctions de transformation industrielle de la matière ligneuse. Permettez-nous, à ce stade-ci, de souligner l'importance d'un programme d'information et de sensibilisation du public afin que ce dernier comprenne adéquatement la notion d'aménagement polyvalent et intégré du territoire et que cette notion ne soit pas confondue avec celle d'aménagement exclusif.

\section{Utilisation des phytocides}

En somme, nous suggérons dans le but de maintenir la capacité de production des usines de transformation, de maximiser les mesures d'aménagement forestier sur les territoires à vocation forestière. À court terme, ces mesures doivent comprendre notamment l'utilisation de phytocides, nos pratiques forestières du passé nous léguant en héritage une forêt dégradée. Cette situation oblige les gestionnaires forestiers à avoir recours à des méthodes artificielles de régénération qui diminuent la variabilité écologique de milieu.

Dans cet optique, le Conseil est d'accord avec l'utilisation de phytocides, principalement le glyphosate permettant le dégagement des plants reboisés où cela est nécessaire car il s'agit d'un produit ayant une très faible résilience dans le milieu. De plus, il constitue un outil à la disposition des forestiers qui a fait ses preuves. Selon le niveau de nos connaissances actuelles, l'utilisation du glyphosate demeure intéressante en raison de sa faible toxicité. En effet, des travaux récents démontrent que la caféine est de 20 fois plus toxique que le glyphosate. 
Nous sommes d'accord pour en réglementer correctement l'utilisation afin que toutes les mesures préventives soient prises afin d'éviter la dégradation de l'environnement et de réduire les risques nocifs pour la santé de l'opérateur. Toutefois, nous entérinons cette pratique dans le seul et unique but de pallier à une dégradation de nos forêts résultant de nos méthodes de gestion passée et tant que d'autres outils n'auront pas été éprouvés. La foresterie de l'avenir doit tendre vers la diminution et l'élimination progressive de ces produits dans les meilleurs délais.

Le développement de méthodes alternatives aux phytocides et compétitives en terme de coût exige beaucoup d'efforts et surtout du temps. Pour ces raisons, le Conseil met l'emphase sur l'évaluation quinquennale de la stratégie de protection qui pourra estimer si l'objectif d'élimination des phytocides d'ici 10 ans est réalisable.

\section{Rôles du professionnel forestier}

Les professionnels forestiers jouent un rôle important en tant que gestionnaire d'une ressource appartenant en majeure partie à l'État et par extension, au public. Ces professionnels doivent donc gérer la forêt en fonction des attentes du public et de l'utilisation qu'on veut tirer de la forêt.

Cette gestion doit se pratiquer autant sur une «table à dessin" que "sur le terrain». L'élaboration d'outils utiles pour le forestier doit demeurer une priorité. Mentionnons qu'il est préférable de confectionner un cadre général de travail assorti de guides pratique plutôt que de consentir à mettre des efforts sur une cartographie complexe, coûteuse et mal utilisée par l'ensemble des utilisateurs.

Les récents échanges à l'intérieur de la revue L'Aubelle, publiée par l'Ordre des ingénieurs forestiers du Québec démontre que la classification écologique fait encore l'objet de grands débats entre écologistes. Il demeure intéressant d'assister à ces discours. Cependant, les forestiers désirent avant tout avoir en leur possession des instruments fiables de travail que ont fait leur preuve. Ce sont des aménagistes qui doivent disposer d'outils adéquats qu'ils sauront utiliser convenablement.

À cet effet, la section Champlain est d'accord pour que la cartographie écologique consiste à produire un document permanent des caractéristiques stables du milieu et que chaque unité doit être identifiée de façon simple et claire. L'aménagiste forestier peut alors prendre les décisions qui s'imposent à l'aide d'un guide d'utilisation ou d'un manuel d'interprétation.

On ne doit pas négliger le rôle joué par le professionnel forestier. Utilisé de façon appropriée, les notions que possèdent le professionnel forestier mèneront vraisemblablement à l'application efficace de la stratégie de protection des forêts. Dans cette ligne de pensée, l'Institut Forestier du Canada collabore avec la Fédération canadienne des associations de professionnels forestiers pour l'élaboration d'un Code national de pratiques forestières. Ce Code permettra aux ingénieurs forestiers de respecter un code d'éthique lui permettant de mener ses activités dans un optique de développement durable. La section Champlain considère que ce Code constitue un outil supplémentaire pour les professionnels.

\section{Recherche et développement}

Les forêts du Québec et l'industrie forestière ont la possibilité de continuer à jouer un rôle essentiel dans notre économie, mais cette possibilité dépend ultimement de la compétitivité de nos produits forestiers sur les marchés mondiaux. La capacité de l'industrie à manufacturer des produits compétitifs dépend du maintien des approvisionnements économiques et soutenus de bois. Ceci signifie qu'à la fois dans la solution des problèmes et à la fois dans le développement de méthodes innovatrices et plus efficaces en terme de coûts, on doit compter sur un haut niveau de recherche. Les programmes de recherche devraient être bien financés à long terme et devraient prendre en considération les besoins de tous les utilisateurs. Ils doivent être dirigés vers l'acquisition des connaissances et des technologies requises pour continuer à générer économiquement l'activité industrielle, et des bénéfices sociaux non-industriels tirés de l'aménagement des forêts et de la vente des produits forestiers.

Nous croyons cependant que dans l'état actuel de nos connaissances en foresterie, il serait peu possible d'avoir en main toutes les informations nécessaires pour aménager nos forêts de façon à maximiser leurs potentiels d'auto-protection. Le texte même de la stratégie fait état à plusieurs reprises de mise en garde relativement au peu de connaissances cumulées dans certains domaines ou encore du besoin d'approfondir les recherches pour d'autres secteurs.

Nous sommes conscients que l'écosystème forestier est d'une complexité telle que la connaissance des interactions entre ses composantes pourra prendre au moins une rotation avant de pouvoir agir sur ces composantes. Compte tenu qu'une rotation correspond à une période de 60 à 100 ans, nous jugeons prématuré à ce moment de vouloir déterminer dans le temps, l'arrêt ou la mise en place de certaines pratiques d'aménagement forestier.

L'avantage de maximiser les mesures d'aménagement forestier au moyen de plantations d'arbres génétiquement améliorés, de méthodes de répression de la végétation indésirable et de contrôle des ravageurs sera dans un premier temps d'augmenter le rendement du milieu mais aussi de réduire dans d'autres zones l'obligation d'utiliser certains territoires à des fins exclusives de production ligneuse.

Il est généralement reconnu qu'un pays industrialisé qui désire demeurer à la fine pointe se doit d'investir au strict minimum, l'équivalent de $1 \%$ de la valeur des livraisons dans la recherche et le développement. Il est également reconnu que le Canada fait office de pays sous-développé en cette matière. Le secteur forestier québécois ne fait pas exception.

En poursuivant cette même logique et sachant que la valeur des livraisons se situe à environ 1,5 milliards $\$$, l'industrie devrait investir 150 millions \$ pour seulement conserver sa situation technologique face aux principaux concurrents. Or, selon les données présentement disponibles (annexe 1), les dépenses actuelles en recherche et développement se chiffrent aux alentours de $80000000 \$$ par année, soit $0.5 \%$ de la valeur des livraisons. De ce pourcentage, la moitié sont investis par les gouvernements, ce qui ramène la part de l'industrie à $0.25 \%$.

Selon l'avis général des économistes spécialisés en la matière, une industrie qui investit si peu se dirige tout droit dans un cul-de-sac. Quelque soit la source des deniers servant à la recherche et au développement, l'industrie forestière, exprimée au sens large, se doit de prendre les moyens nécessaires pour pallier à ce manque. Plus précisément, l'industrie forestière, exprimée au sens strict, doit faire sa part pour demeurer compétitive. En augmentant sa contribution fainancière, l'industrie forestière peut alors accroître sa 
gestion des programmes de recherche et développement, le secteur privé s'étant toujours montré plus efficace et efficient que l'État.

La section Champlain croit que pour répondre aux attentes de la stratégie de protection des forêts, des efforts importants devront être consentis en matière de connaissance des traitements sylvicoles, la transformation de toutes les essences commerciales, la formation d'ouvriers sylvicoles, le transfert technologique aux professionnels forestiers et la mise en place d'un programme d'information et de sensibilisation du public.

Il est quelque peu désolant que la stratégie de protection des forêts, telle qu'elle a été présentée, consacre seulement 1,5 page à la recherche et la formation de la main-d'oeuvre.

\section{Conclusion}

Le secteur forestier québécois est au seuil d'une nouvelle ère d'utilisation des ressources forestières. Dorénavant, les forêts ne seront plus seulement comptabilisées en terme de matière ligneuse mais aussi en tant que ressources faunique, récréative et touristique, tout en maximisant ses effets de régulateur climatologique.

La stratégie de protection des forêts ouvre timidement la porte sur ces possibilités. Cependant, nous sommes certains que les forestiers professionnels constituent le noyau de personnes qui peuvent le mieux réaliser les attentes du public tout en permettant au secteur forestier de conserver et de développer sa vitalité, à condition que des efforts en matière de recherche forestière soient consentis par tous et chacun.

贾

Annexe

COMPILATION DES INFORMATIONS RELATIVES AUX DÉPENSES DE R ET D DANS LE DOMAINE FORESTIER EN 1988-89 (DÉTAILLÉ)

\begin{tabular}{|l|c|c|}
\hline & $\begin{array}{c}\text { DÉPENSES } \\
\text { DE R ET D } \\
\text { THĖMES DE R ET D }\end{array}$ & $\%$ \\
\hline $\begin{array}{l}\text { Écologie, rendement et utilisation } \\
\text { polyvalent }\end{array}$ & 4,845 & 6.0 \\
\hline Amélioration des forêts & 19,125 & 23.5 \\
\hline Protection des forêts & 10,995 & 13.5 \\
\hline Récolte et transport & 3,650 & 4.5 \\
\hline Pâtes et papiers & 31,885 & 39.2 \\
\hline Sciage & 2,480 & 3.0 \\
\hline Panneaux & 1,160 & 1.5 \\
\hline Produits autres & 2,235 & 2.7 \\
\hline Transfer de technologie & 4,975 & 6.1 \\
\hline TOTAL & 81,350 & $100 \%$ \\
\hline
\end{tabular}

EFFORTS DE R ET D FORESTIER

\begin{tabular}{|l|r|r|}
\hline \multicolumn{1}{|c|}{ ACTIVITÉS } & 000\$ & \% \\
\hline Ressources forestière & 34,965 & 43 \\
\hline Récolte et transport & 3,650 & 4 \\
\hline Transformation & 37,760 & 46 \\
\hline Transfert de technologie & 4,975 & 6 \\
\hline TOTAL & 81,350 & 100 \\
\hline
\end{tabular}

EFFORTS DE R ET D FORESTIER EN FONCTION D'INDICATEURS ÉCONOMIQUES

\begin{tabular}{|l|c|c|}
\hline \multicolumn{1}{|c|}{ INDICATEURS } & SECTEUR FORESTIER & $\begin{array}{c}\text { TOUS SECTEURS } \\
\text { (QUÉBEC) }\end{array}$ \\
\hline$\%$ du PIB & $1.3 \%$ & $1.3 \%$ \\
\hline$\%$ des livraisons & $0.6 \%$ & \\
\hline Par $\mathrm{m}^{3}$ de bois récolté & $225 \$$ & \\
\hline $\begin{array}{l}\text { Par Ha de forêt } \\
\text { productive }\end{array}$ & $410 \$$ & \\
\hline
\end{tabular}

FINANCEMENT DE LA RECHERCHE FORESTIÈRE AU QUÉBEC

\begin{tabular}{|l|c|}
\hline PROVENANCE DES FONDS & $\%$ \\
\hline Gouvernement du Québec & 26 \\
\hline Gouvernement du Canada & 20.5 \\
\hline Secteur privé & 53,3 \\
\hline
\end{tabular}

Source: Conseil de la recherche forestière du Québec 